Dr. Dirk Heinrich, Bundesvorsitzender des NAV-Virchow-Bundes, meinte, dass die Praxisgebühr dringend abgeschafft werden müsse. Das würde die Ärzte enorm entlasten. Bereits im März hatte die KBV darauf verwiesen, dass jeder niedergelassene Arzt mit seinem Praxisteam jährlich rund 120 Stunden aufwende, um die Praxisgebühr einzubehalten und zu quittieren.

\section{Beruf und Privatleben sind nur eingeschränkt vereinbar}

Unzufrieden sind Ärzte zudem mit der Vereinbarkeit von Familie und Beruf. „Die Mehrzahl sieht diese Bereiche als lediglich eingeschränkt vereinbar", so Köhler. Insgesamt haben der Umfrage zufolge $64 \%$ nicht genügend Zeit für persönliche Interessen. Die Pläne für private Aktivitäten müssten zudem oft geändert werden, weil berufliche Verpflichtungen dazwischenfunken (26\%). 17 sagten sogar, dass die Anforderungen der Arbeit das Privat- und Familienleben „stören“.

Heinrich forderte Konsequenzen aus den Umfrageergebnissen: „Die Ärzte arbeiten an der Belastungsgrenze." Daher müsse man von den hohen Fallzahlen in den Praxen wegkommen.

\section{Kassen unterstellen zeitlichen} Spielraum für mehr Sprechzeiten

Der Spitzenverband der GKV beurteilte den Ärztemonitor kritisch: Ärzte könnten leicht mehr Zeit für ihre Patienten erhalten, sagte Florian Lanz, Sprecher des Verbandes. Der Verband habe im Jahr 2009 selbst eine Umfrage beim Marktforschungsinstitut FORSA (Gesellschaft für Sozialforschung und statistische Analyse in Auftrag) gegeben. Demnach wenden niedergelassene Haus- und Fachärzte im Schnitt 28,5 Stunden pro Woche für ihre Sprechstunden auf. Rechne man Termine nach Vereinbarung hinzu, kämen Fachärzte auf knapp 32 Stunden, die sie im Durchschnitt ihren Patienten zur Verfügung stünden. Die große Mehrheit der Hausärzte biete darüber hinaus Hausbesuche an und erreicht so durchschnittlich 36 Stunden. „Den zeitlichen Spielraum für mehr Sprechzeiten scheint es auf jeden Fall zu geben“, so Lanz.

Sunna Gieseke

Kassen erteilen Ärzten eine Abfuhr

Der GKV-Spitzenverband hat die Forderungen der KBV von etwa 3,5 Milliarden Euro mehr für den Honorartopf der Ärzte als überzogen zurückgewiesen. Die zurzeit komfortablen Finanzreserven seien kein Grund für solche Forderungen, meinte Dr. Doris Pfeiffer, Vorsitzende des GKV-Spitzenverbandes. Insgesamt etwa eine Milliarde Euro mehr für die Arzthonorare im Jahr 2013 seien realistisch. Für 2012 könne man sich auf 600 Millionen Euro einigen. KBV-Chef Dr. Andreas Köhler widersprach vehement: „Der Gesetzgeber sieht ganz klar vor, dass die Mehrkosten, die durch die steigende Krankheitslast in der Bevölkerung entstehen, von der Krankenversicherung finanziert werden müssen." Die Honorarforderungen der Ärzte seien somit „berechtigt" und beruhten auf einer gesetzlichen Grundlage.

\title{
Krankenversicherung bunkert Milliarden
}

Die Kassen sind bis zum Rand voll, die Prognosen sehr gut: Bis zum Jahresende sollen sogar noch weitere sieben Milliarden Euro mehr im Topf sein. Also doch Prämien für die Versicherten? Der GKV-Spitzenverband sieht dafür keinen Spielraum.

$D_{k}^{3}$ as Finanzpolster der gesetzlichen Krankenversicherung beträgt zurzeit etwa 19,5 Milliarden Euro - Tendenz steigend. Trotz der Rekordüberschüsse im Gesundheitssystem wollen die Kassen jedoch das Geld horten. Es sei eben nicht so reichlich, „dass man nun das Geld zum Fenster rauswerfen kann“, so Dr. Doris Pfeiffer, Vorsitzende des GKV-Spitzenverbandes. Koalitionsinterne Prognosen besagen jedoch, dass die Überschüsse im System der gesetzlichen Krankenversicherung in diesem Jahr um sieben Milliarden auf dann sogar knapp 27 Milliarden Euro steigen werden. Die genaue Zahl wollte Pfeiffer nicht bestätigen. Allerdings: Fest stehe schon jetzt, dass das Finanzpolster weiter wachse, so Pfeiffer.

\section{FDP will Prämienzahlungen} an die Versicherten erzwingen

Gesundheitsminister Daniel Bahr (FDP) drängt die Kassen aufgrund der guten finanziellen Lage bereits seit Februar dieses Jahres, einen Teil des Geldes als Prämie an die Mitglieder auszuschütten - bisher mit mäßigem Erfolg. Im Mai setzte Bahr dann noch eins drauf: Notfalls wolle er die Kassen per Gesetz zu Prämienzahlungen zwingen. Pfeiffer zeigt sich angesichts dieser Drohung unbeeindruckt: Die düsteren Wolken am Finanzhimmel ermöglichten eben nicht genügend Spielraum, um das Geld zu diesem Zeitpunkt an die Versicherten zu verteilen. Der GKV-Spitzenverband erwarte Ausgabesteigerungen für Arzneimittel sowie die ambulante und statio- näre Versorgung von fast zehn Milliarden Euro in den kommenden beiden Jahren. Zudem gebe es konjunkturelle Risiken im Euroraum, meinte Pfeiffer.

Auch das Bundesversicherungsamt (BVA) hatte bereits drei Kassen aufgefordert, angesichts hoher Rücklagen eine Prämienzahlung zu prüfen. Diese Nachricht erhielten die Techniker Krankenkasse (TK), die IKK gesundplus und die Hanseatische Krankenkasse. Die TK hat das Amt um Aufschub bis Ende Juni gebeten. Doch das BVA bleibt dabei: „Krankenkassen sind keine Sparkassen. “Diese seien aufgefordert, ihren Patienten Prämien auszuschütten oder die Versorgung zu verbessern.

\section{CDU will Rücklagen der Kassen} für schlechtere Zeiten bewahren

Seit Anfang 2009 gilt ein bundesweit einheitlicher Satz von $15,5 \%$. Eine allgemeine Beitragssatzerhöhung oder $\mathrm{Zu}$ satzbeiträge einzelner Kassen schloss Pfeiffer bis Ende 2013 - dem Jahr der nächsten Bundestagswahl - aus. Doch gerade im Wahljahr müssen die Kassen die Milliarden gegen weitere Zugriffe verteidigen: „Unsere Sorge ist, dass die durchaus komfortable Finanzlage die Politik dazu verführen könnte, besonders großzügig zu sein." Aus Sicht von CDU-Politiker Jens Spahn ist das reine Panikmache der Kassen: „Die Koalition wird mit der nötigen Umsicht mit den Rücklagen der Kassen umgehen“, sagte er. Es gehe vor allem darum, die Rücklagen für schlechtere Zeiten zu bewahren.

Sunna Gieseke 